Acta Crystallographica Section E

Structure Reports

Online

ISSN 1600-5368

\section{4-Chloro- $\mathrm{N}$-(3-methylbenzoyl)benzene- sulfonamide monohydrate}

\section{P. A. Suchetan, ${ }^{\text {a Sabine Foro, }},{ }^{b}$ B. Thimme Gowda ${ }^{a}$ and M. Shet Prakash ${ }^{\mathrm{c}}$}

a Department of Chemistry, Mangalore University, Mangalagangotri 574199 Mangalore, India, ${ }^{\mathbf{b}}$ Institute of Materials Science, Darmstadt University of Technology, Petersenstrasse 23, D-64287 Darmstadt, Germany, and ${ }^{\mathbf{c}}$ Department of Chemistry, University College of Science, Tumkur University, Tumkur 572 102, India Correspondence e-mail: gowdabt@yahoo.com

Received 29 November 2011; accepted 1 December 2011

Key indicators: single-crystal X-ray study; $T=293 \mathrm{~K}$; mean $\sigma(\mathrm{C}-\mathrm{C})=0.010 \AA$; $R$ factor $=0.105 ; w R$ factor $=0.185 ;$ data-to-parameter ratio $=13.4$.

In the title compound, $\mathrm{C}_{14} \mathrm{H}_{12} \mathrm{ClNO}_{3} \mathrm{~S} \cdot \mathrm{H}_{2} \mathrm{O}$, the dihedral angle between the sulfonyl and benzoyl benzene rings is $84.4(2)^{\circ}$. In the crystal, every water molecule forms four hydrogen bonds with three different molecules of 4-chloro- $\mathrm{N}$-(3-methylbenzoyl)benzenesulfonamide. One of the water $\mathrm{H}$ atoms forms a bifurcated hydrogen bond with both the sulfonyl and the carbonyl $\mathrm{O}$ atoms of the same molecule. Molecules are linked into layers in the $a b$ plane through $\mathrm{N}-\mathrm{H} \cdots \mathrm{O}$ and $\mathrm{O}-\mathrm{H} \cdots \mathrm{O}$ hydrogen bonds.

\section{Related literature}

For our studies on the effects of substituents on the structures and other aspects of $N$-(aryl)-amides, see: Gowda et al. (2004), on $N$-(aryl)-methanesulfonamides, see: Jayalakshmi \& Gowda (2004), on $N$-(aryl)-arylsulfonamides, see: Gowda et al. (2003), on $N$-(substitutedbenzoyl)-arylsulfonamides, see: Suchetan et al. (2011) and on $N$-chloroarylamides, see: Gowda et al. (1996).

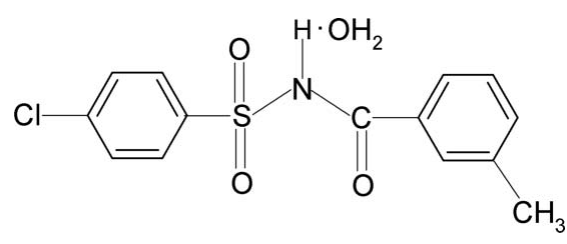

\section{Experimental}

Crystal data

$\mathrm{C}_{14} \mathrm{H}_{12} \mathrm{ClNO}_{3} \mathrm{~S} \cdot \mathrm{H}_{2} \mathrm{O}$

$M_{r}=327.77$

Orthorhombic, $\mathrm{Pbca}$ $a=5.0148(6) \AA$ $b=12.864(2) \AA$

$c=46.314(5) \AA$

$V=2987.7(7) \AA^{3}$

$Z=8$

Mo $K \alpha$ radiation

$\mu=0.41 \mathrm{~mm}^{-1}$

$T=293 \mathrm{~K}$

$0.46 \times 0.14 \times 0.06 \mathrm{~mm}$

Data collection

Oxford Diffraction Xcalibur diffractometer with a Sapphire CCD detector

Absorption correction: multi-scan (CrysAlis RED; Oxford

Diffraction, 2009)

$T_{\text {min }}=0.834, T_{\max }=0.976$ 5972 measured reflections 2684 independent reflections 1959 reflections with $I>2 \sigma(I)$ $R_{\text {int }}=0.037$

Refinement

$R\left[F^{2}>2 \sigma\left(F^{2}\right)\right]=0.105$

$w R\left(F^{2}\right)=0.185$

$S=1.35$

2684 reflections

200 parameters

3 restraints

$\mathrm{H}$ atoms treated by a mixture of independent and constrained refinement

$\Delta \rho_{\max }=0.33{\mathrm{e} \AA^{-3}}^{-3}$

$\Delta \rho_{\min }=-0.34$ e $\AA^{-3}$

Table 1

Hydrogen-bond geometry $\left(\AA{ }^{\circ}\right)$.

\begin{tabular}{lllll}
\hline$D-\mathrm{H} \cdots A$ & $D-\mathrm{H}$ & $\mathrm{H} \cdots A$ & $D \cdots A$ & $D-\mathrm{H} \cdots A$ \\
\hline $\mathrm{N} 1-\mathrm{H} 1 N \cdots \mathrm{O} 4$ & $0.86(2)$ & $1.93(2)$ & $2.771(8)$ & $169(6)$ \\
$\mathrm{O} 4-\mathrm{H} 41 \cdots 1^{\mathrm{i}}$ & $0.84(2)$ & $2.29(7)$ & $2.916(7)$ & $131(8)$ \\
$\mathrm{O} 4-\mathrm{H} 41 \cdots \mathrm{O}^{\mathrm{i}}$ & $0.84(2)$ & $2.42(6)$ & $3.117(8)$ & $140(8)$ \\
$\mathrm{O}^{\mathrm{i}}-\mathrm{H} 42 \cdots \mathrm{O} 2^{\mathrm{ii}}$ & $0.84(2)$ & $2.35(6)$ & $3.022(8)$ & $137(8)$ \\
\hline
\end{tabular}

Symmetry codes: (i) $-x+\frac{1}{2}, y+\frac{1}{2}, z$; (ii) $x+1, y, z$.

Data collection: CrysAlis CCD (Oxford Diffraction, 2009); cell refinement: CrysAlis RED (Oxford Diffraction, 2009); data reduction: CrysAlis RED; program(s) used to solve structure: SHELXS97 (Sheldrick, 2008); program(s) used to refine structure: SHELXL97 (Sheldrick, 2008); molecular graphics: PLATON (Spek, 2009); software used to prepare material for publication: SHELXL97.

BTG thanks the University Grants Commission, Government of India, New Delhi, for a special grant under the UGCBSR one-time grant to faculty.

Supplementary data and figures for this paper are available from the IUCr electronic archives (Reference: BT5739).

\title{
References
}

Gowda, B. T., Dou, S. Q. \& Weiss, A. (1996). Z. Naturforsch. Teil A, 51, 627 636.

Gowda, B. T., Jyothi, K., Kozisek, J. \& Fuess, H. (2003). Z. Naturforsch. Teil A, 58, 656-660.

Gowda, B. T., Svoboda, I. \& Fuess, H. (2004). Z. Naturforsch. Teil A, 59, 845852.

Jayalakshmi, K. L. \& Gowda, B. T. (2004). Z. Naturforsch. Teil A, 59, 491-500. Oxford Diffraction (2009). CrysAlis CCD and CrysAlis RED. Oxford Diffraction Ltd, Yarnton, England.

Sheldrick, G. M. (2008). Acta Cryst. A64, 112-122.

Spek, A. L. (2009). Acta Cryst. D65, 148-155.

Suchetan, P. A., Foro, S. \& Gowda, B. T. (2011). Acta Cryst. E67, o22. 


\section{supporting information}

Acta Cryst. (2012). E68, o46 [doi:10.1107/S1600536811051932]

\section{4-Chloro-N-(3-methylbenzoyl)benzenesulfonamide monohydrate}

\section{P. A. Suchetan, Sabine Foro, B. Thimme Gowda and M. Shet Prakash}

\section{S1. Comment}

Diaryl acylsulfonamides are known as potent antitumor agents. As part of our studies on the substituent effects on the structures and other aspects of $N$-(aryl)-amides (Gowda et al., 2004), $N$-(aryl)-methanesulfonamides (Jayalakshmi \& Gowda, 2004), $N$-(aryl)-arylsulfonamides (Gowda et al., 2003); $N$-(substitutedbenzoyl)-arylsulfonamides (Suchetan et al., 2011) and $N$-chloro-arylsulfonamides (Gowda et al., 1996), in the present work, the crystal structure of 4-Chloro$\mathrm{N}$-(3-methylbenzoyl)-benzenesulfonamide monohydrate (I) has been determined (Fig.1).

The conformations of the $\mathrm{N}-\mathrm{H}$ and $\mathrm{C}=\mathrm{O}$ bonds in the $\mathrm{C}-\mathrm{SO}_{2}-\mathrm{NH}-\mathrm{C}(\mathrm{O})$ segment are anti to each other (Fig.1), similar to that observed in 4-Chloro- $N$-(2-methylbenzoyl)-benzenesulfonamide monohydrate (II) (Suchetan et al., 2011). The molecule is twisted at the $S$ - atom with the torsional angle of $-70.67(55)^{\circ}$, compared to the value of $-69.2(2)^{\circ}$ in (II).

The dihedral angle between the sulfonyl benzene ring and the $-\mathrm{SO}_{2}-\mathrm{NH}-\mathrm{C}-\mathrm{O}$ segment is $78.4(2)^{\circ}$, compared to the value of $87.2(1)^{\circ}$ in (II). Furthermore, the dihedral angle between the sulfonyl and the benzoyl benzene rings is $84.4(2)^{\circ}$, compared to the value of $57.7(1)^{\circ}$ in (II).

Further, the crystal structure shows interesting H-bonding. Every water molecule forms four H-bonds with three different molecules of the title compound. One of the $\mathrm{H}$-atoms of the water molecule forms simultaneous H-bonding with both the sulfonyl and the carbonyl oxygen atoms of the same molecule.

The packing of molecules through $\mathrm{N} 1-\mathrm{H} 1 \mathrm{~N} \cdots \mathrm{O} 4, \mathrm{O} 4-\mathrm{H} 41 \cdots \mathrm{O} 1, \mathrm{O} 4-\mathrm{H} 41 \cdots \mathrm{O} 3$ and $\mathrm{O} 4-\mathrm{H} 42 \cdots \mathrm{O} 2$ hydrogen bonds (Table 1) is shown in Fig. 2.

\section{S2. Experimental}

The title compound was prepared by refluxing a mixture of $m$-methyl benzoic acid ( 0.02 mole), 4-chlorobenzenesulfonamide ( 0.02 mole) and excess phosphorous oxy chloride for $3 \mathrm{~h}$ on a water bath. The resultant mixture was cooled and poured into crushed ice. The solid, 4-Chloro- $N$-(3-methylbenzoyl)-benzenesulfonamide monohydrate, obtained was filtered, washed thoroughly with water and then dissolved in sodium bicarbonate solution. The compound was later reprecipitated by acidifying the filtered solution with dilute $\mathrm{HCl}$. It was filtered, dried and recrystallized.

Needle like colourless single crystals of the title compound used in X-ray diffraction studies were obtained by slow evaporation of its ethanol-tetrahydrofuran solution at room temperature.

\section{S3. Refinement}

The $\mathrm{H}$ atoms of the $\mathrm{NH}$ group and of the water molecule were located in a difference map and later restrained to $\mathrm{N}-\mathrm{H}=$ 0.86 (2) $\AA$ and $\mathrm{O}-\mathrm{H}=0.85$ (2) $\AA$. The other $\mathrm{H}$ atoms were positioned with idealized geometry using a riding model with the aromatic $\mathrm{C}-\mathrm{H}=0.93 \AA$ and methyl $\mathrm{C}-\mathrm{H}=0.96 \AA$. All $\mathrm{H}$ atoms were refined with isotropic displacement parameters set to 1.2 times of the $U_{\mathrm{eq}}$ of the parent atom. 


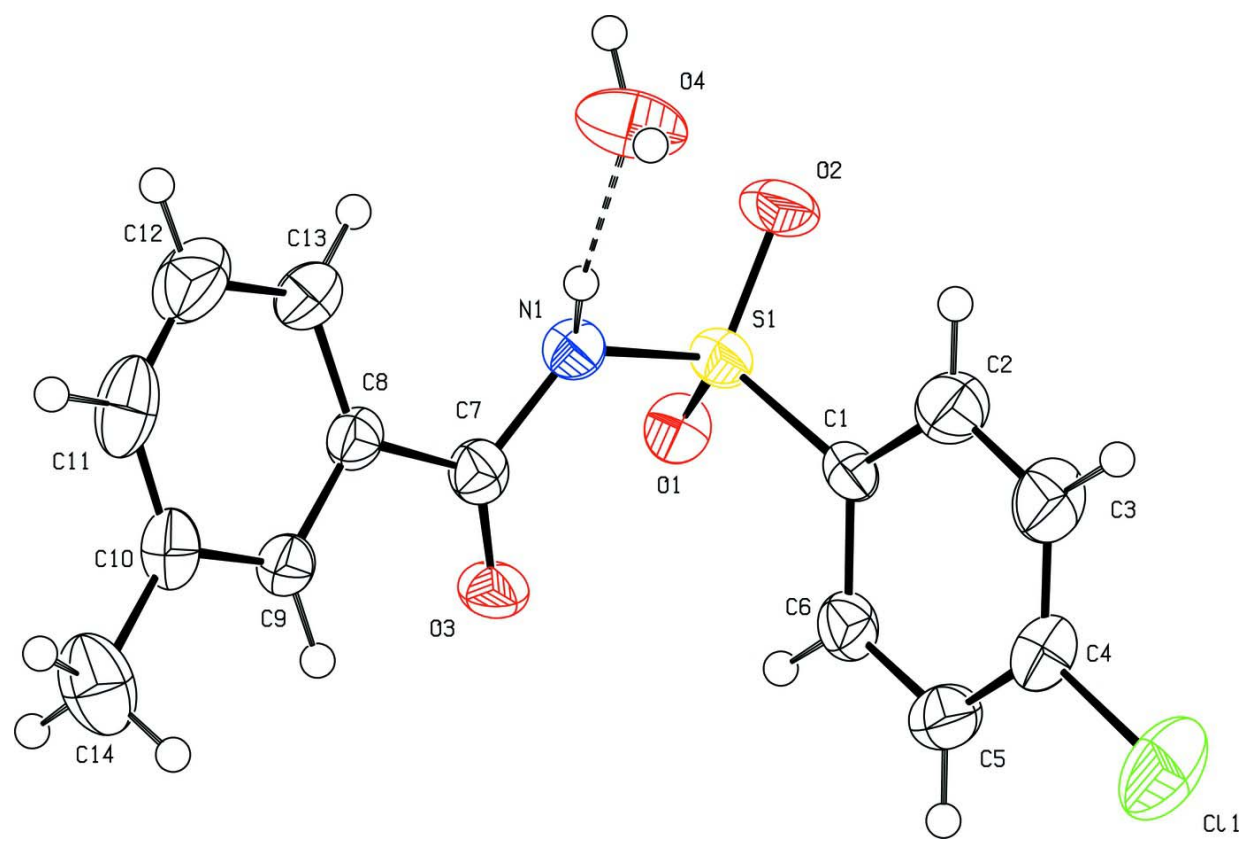

\section{Figure 1}

Molecular structure of the title compound, showing the atom-labelling scheme. Displacement ellipsoids are drawn at the $50 \%$ probability level.

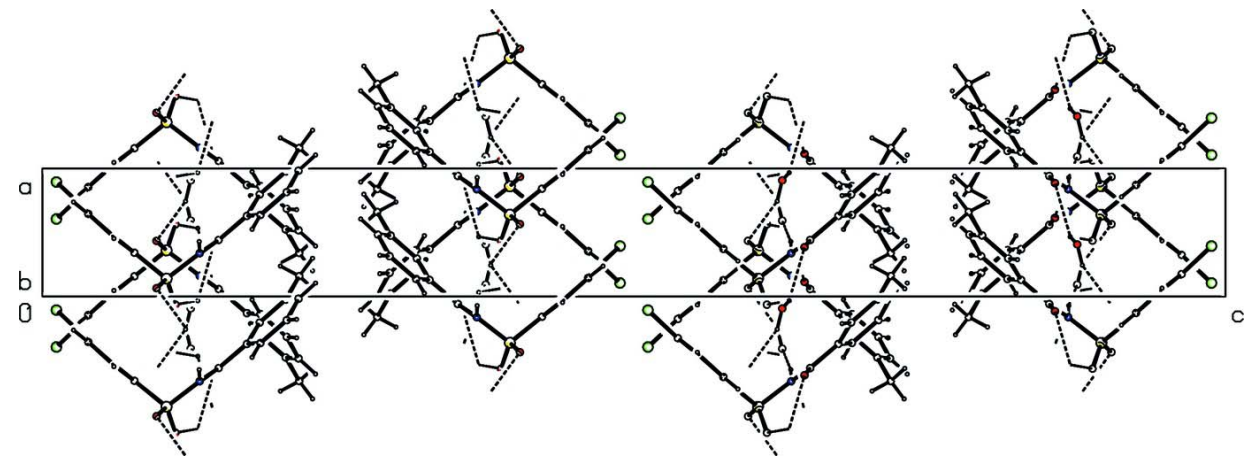

Figure 2

Molecular packing in the title compound. Hydrogen bonds are shown as dashed lines.

\section{4-Chloro- $N$-(3-methylbenzoyl)benzenesulfonamide monohydrate}

\section{Crystal data}

$\mathrm{C}_{14} \mathrm{H}_{12} \mathrm{ClNO}_{3} \mathrm{~S} \cdot \mathrm{H}_{2} \mathrm{O}$

$M_{r}=327.77$

Orthorhombic, $\mathrm{Pbca}$

Hall symbol: -P 2ac 2ab

$a=5.0148$ (6) $\AA$

$b=12.864$ (2) $\AA$

$c=46.314(5) \AA$

$V=2987.7(7) \AA^{3}$

$Z=8$
$F(000)=1360$

$D_{\mathrm{x}}=1.457 \mathrm{Mg} \mathrm{m}^{-3}$

Mo $K \alpha$ radiation, $\lambda=0.71073 \AA$

Cell parameters from 1913 reflections

$\theta=2.6-27.8^{\circ}$

$\mu=0.41 \mathrm{~mm}^{-1}$

$T=293 \mathrm{~K}$

Needle, colourless

$0.46 \times 0.14 \times 0.06 \mathrm{~mm}$ 


\section{Data collection}

Oxford Diffraction Xcalibur

diffractometer with a Sapphire CCD detector

Radiation source: fine-focus sealed tube

Graphite monochromator

Rotation method data acquisition using $\omega$ and phi scans

Absorption correction: multi-scan

(CrysAlis RED; Oxford Diffraction, 2009)

$T_{\min }=0.834, T_{\max }=0.976$

\section{Refinement}

Refinement on $F^{2}$

Least-squares matrix: full

$R\left[F^{2}>2 \sigma\left(F^{2}\right)\right]=0.105$

$w R\left(F^{2}\right)=0.185$

$S=1.35$

2684 reflections

200 parameters

3 restraints

Primary atom site location: structure-invariant direct methods
5972 measured reflections

2684 independent reflections

1959 reflections with $I>2 \sigma(I)$

$R_{\text {int }}=0.037$

$\theta_{\max }=25.4^{\circ}, \theta_{\min }=2.6^{\circ}$

$h=-3 \rightarrow 6$

$k=-9 \rightarrow 15$

$l=-55 \rightarrow 55$
Secondary atom site location: difference Fourier map

Hydrogen site location: inferred from neighbouring sites

$\mathrm{H}$ atoms treated by a mixture of independent and constrained refinement

$w=1 /\left[\sigma^{2}\left(F_{\mathrm{o}}^{2}\right)+(0 . P)^{2}+17.7362 P\right]$

where $P=\left(F_{\mathrm{o}}^{2}+2 F_{\mathrm{c}}^{2}\right) / 3$

$(\Delta / \sigma)_{\max }=0.001$

$\Delta \rho_{\max }=0.33$ e $\AA^{-3}$

$\Delta \rho_{\text {min }}=-0.34$ e $\AA^{-3}$

\section{Special details}

Experimental. CrysAlis RED (Oxford Diffraction, 2009) Empirical absorption correction using spherical harmonics, implemented in SCALE3 ABSPACK scaling algorithm.

Geometry. All e.s.d.'s (except the e.s.d. in the dihedral angle between two 1.s. planes) are estimated using the full covariance matrix. The cell e.s.d.'s are taken into account individually in the estimation of e.s.d.'s in distances, angles and torsion angles; correlations between e.s.d.'s in cell parameters are only used when they are defined by crystal symmetry. An approximate (isotropic) treatment of cell e.s.d.'s is used for estimating e.s.d.'s involving 1.s. planes.

Refinement. Refinement of $F^{2}$ against ALL reflections. The weighted $R$-factor $w R$ and goodness of fit $S$ are based on $F^{2}$, conventional $R$-factors $R$ are based on $F$, with $F$ set to zero for negative $F^{2}$. The threshold expression of $F^{2}>\sigma\left(F^{2}\right)$ is used only for calculating $R$-factors(gt) etc. and is not relevant to the choice of reflections for refinement. $R$-factors based on $F^{2}$ are statistically about twice as large as those based on $F$, and $R$ - factors based on ALL data will be even larger.

Fractional atomic coordinates and isotropic or equivalent isotropic displacement parameters $\left(\AA^{2}\right)$

\begin{tabular}{lllll}
\hline & $x$ & $y$ & $z$ & $U_{\text {iso }} * / U_{\text {eq }}$ \\
\hline C1 & $0.3419(12)$ & $-0.0253(5)$ & $0.07777(12)$ & $0.0283(14)$ \\
C2 & $0.4759(15)$ & $0.0400(6)$ & $0.05920(14)$ & $0.0469(19)$ \\
H2 & 0.4533 & 0.1117 & 0.0604 & $0.056^{*}$ \\
C3 & $0.6448(16)$ & $-0.0027(6)$ & $0.03877(15)$ & $0.051(2)$ \\
H3 & 0.7378 & 0.0403 & 0.0262 & $0.061^{*}$ \\
C4 & $0.6751(15)$ & $-0.1085(6)$ & $0.03706(14)$ & $0.0423(18)$ \\
C5 & $0.5420(15)$ & $-0.1736(6)$ & $0.05504(14)$ & $0.0437(18)$ \\
H5 & 0.5650 & -0.2451 & 0.0536 & $0.052^{*}$ \\
C6 & $0.3717(14)$ & $-0.1325(5)$ & $0.07558(13)$ & $0.0367(16)$ \\
H6 & 0.2776 & -0.1764 & 0.0879 & $0.044 *$ \\
C7 & $0.4414(13)$ & $-0.0406(5)$ & $0.14890(13)$ & $0.0323(15)$ \\
C8 & $0.6298(13)$ & $-0.0066(5)$ & $0.17281(13)$ & $0.0308(15)$ \\
C9 & $0.7972(13)$ & $-0.0844(5)$ & $0.18361(13)$ & $0.0335(16)$
\end{tabular}




$\begin{array}{lllll}\text { H9 } & 0.7884 & -0.1511 & 0.1759 & 0.040^{*} \\ \text { C10 } & 0.9761(14) & -0.0630(6) & 0.20566(14) & 0.0403(18) \\ \text { C11 } & 0.9834(16) & 0.0360(7) & 0.21646(15) & 0.055(2) \\ \text { H11 } & 1.1054 & 0.0517 & 0.2309 & 0.066^{*} \\ \text { C12 } & 0.8156(18) & 0.1131(7) & 0.20656(16) & 0.059(2) \\ \text { H12 } & 0.8217 & 0.1791 & 0.2148 & 0.071^{*} \\ \text { C13 } & 0.6372(16) & 0.0924(5) & 0.18426(14) & 0.0437(18) \\ \text { H13 } & 0.5253 & 0.1441 & 0.1772 & 0.052^{*} \\ \text { C14 } & 1.1601(16) & -0.1458(7) & 0.21706(17) & 0.063(2) \\ \text { H14A } & 1.2381 & -0.1830 & 0.2012 & 0.075^{*} \\ \text { H14B } & 1.0610 & -0.1933 & 0.2289 & 0.075^{*} \\ \text { H14C } & 1.2986 & -0.1141 & 0.2283 & 0.075^{*} \\ \text { N1 } & 0.3383(10) & 0.0427(4) & 0.13292(11) & 0.0302(12) \\ \text { H1N } & 0.429(11) & 0.099(3) & 0.1325(14) & 0.036^{*} \\ \text { O1 } & -0.0632(9) & -0.0465(3) & 0.11239(9) & 0.0360(11) \\ \text { O2 } & 0.0653(10) & 0.1296(3) & 0.09642(10) & 0.0441(13) \\ \text { O3 } & 0.3853(10) & -0.1289(3) & 0.14414(9) & 0.0387(12) \\ \text { O4 } & 0.5930(12) & 0.2319(4) & 0.12531(16) & 0.0649(17) \\ \text { H41 } & 0.533(17) & 0.289(4) & 0.1312(18) & 0.078^{*} \\ \text { H42 } & 0.756(6) & 0.234(7) & 0.1210(18) & 0.078^{*} \\ \text { C11 } & 0.8942(5) & -0.1606(2) & 0.01180(5) & 0.0729(7) \\ \text { S1 } & 0.1393(3) & 0.02651(13) & 0.10489(3) & 0.0315(4)\end{array}$

Atomic displacement parameters $\left(\AA^{2}\right)$

\begin{tabular}{lllllll}
\hline & $U^{11}$ & $U^{22}$ & $U^{33}$ & $U^{12}$ & $U^{13}$ & $U^{23}$ \\
\hline C1 & $0.031(3)$ & $0.027(3)$ & $0.027(3)$ & $-0.002(3)$ & $-0.003(3)$ & $0.006(3)$ \\
C2 & $0.056(5)$ & $0.040(4)$ & $0.045(4)$ & $-0.006(4)$ & $0.008(4)$ & $-0.001(4)$ \\
C3 & $0.059(5)$ & $0.051(5)$ & $0.043(4)$ & $-0.020(4)$ & $0.021(4)$ & $0.001(4)$ \\
C4 & $0.043(4)$ & $0.049(5)$ & $0.035(4)$ & $-0.003(4)$ & $0.004(3)$ & $-0.008(3)$ \\
C5 & $0.053(5)$ & $0.035(4)$ & $0.044(4)$ & $-0.001(4)$ & $0.005(4)$ & $-0.004(3)$ \\
C6 & $0.042(4)$ & $0.037(4)$ & $0.031(3)$ & $-0.005(4)$ & $0.005(3)$ & $0.005(3)$ \\
C7 & $0.030(3)$ & $0.037(4)$ & $0.030(3)$ & $0.002(3)$ & $0.005(3)$ & $0.002(3)$ \\
C8 & $0.035(4)$ & $0.032(4)$ & $0.026(3)$ & $-0.003(3)$ & $0.004(3)$ & $-0.002(3)$ \\
C9 & $0.040(4)$ & $0.032(4)$ & $0.028(3)$ & $0.000(3)$ & $0.004(3)$ & $-0.001(3)$ \\
C10 & $0.036(4)$ & $0.056(5)$ & $0.029(3)$ & $-0.001(4)$ & $0.001(3)$ & $0.002(4)$ \\
C11 & $0.045(5)$ & $0.087(7)$ & $0.033(4)$ & $-0.009(5)$ & $-0.011(4)$ & $-0.010(4)$ \\
C12 & $0.072(6)$ & $0.058(5)$ & $0.048(5)$ & $-0.011(5)$ & $-0.004(5)$ & $-0.017(4)$ \\
C13 & $0.055(5)$ & $0.038(4)$ & $0.038(4)$ & $-0.001(4)$ & $-0.005(4)$ & $-0.009(3)$ \\
C14 & $0.045(5)$ & $0.088(7)$ & $0.055(5)$ & $0.005(5)$ & $-0.008(4)$ & $0.018(5)$ \\
N1 & $0.029(3)$ & $0.028(3)$ & $0.033(3)$ & $-0.006(3)$ & $-0.005(2)$ & $-0.002(2)$ \\
O1 & $0.032(2)$ & $0.039(3)$ & $0.037(2)$ & $-0.006(2)$ & $0.002(2)$ & $-0.001(2)$ \\
O2 & $0.047(3)$ & $0.030(3)$ & $0.055(3)$ & $0.012(2)$ & $-0.009(3)$ & $0.004(2)$ \\
O3 & $0.050(3)$ & $0.023(3)$ & $0.043(3)$ & $-0.004(2)$ & $-0.012(2)$ & $-0.001(2)$ \\
O4 & $0.051(4)$ & $0.036(3)$ & $0.107(5)$ & $-0.003(3)$ & $-0.004(4)$ & $0.009(3)$ \\
C11 & $0.0690(15)$ & $0.0909(17)$ & $0.0590(12)$ & $-0.0093(14)$ & $0.0315(12)$ & $-0.0208(13)$ \\
S1 & $0.0309(8)$ & $0.0296(8)$ & $0.0338(8)$ & $0.0019(8)$ & $-0.0033(7)$ & $0.0031(8)$ \\
& & & & & & \\
\hline
\end{tabular}


Geometric parameters $\left(\AA,{ }^{\circ}\right)$

\begin{tabular}{|c|c|c|c|}
\hline $\mathrm{C} 1-\mathrm{C} 2$ & $1.377(9)$ & C9-H9 & 0.9300 \\
\hline $\mathrm{C} 1-\mathrm{C} 6$ & $1.392(9)$ & $\mathrm{C} 10-\mathrm{C} 11$ & $1.369(10)$ \\
\hline $\mathrm{C} 1-\mathrm{S} 1$ & $1.747(6)$ & $\mathrm{C} 10-\mathrm{C} 14$ & $1.505(10)$ \\
\hline $\mathrm{C} 2-\mathrm{C} 3$ & $1.384(10)$ & $\mathrm{C} 11-\mathrm{C} 12$ & $1.380(11)$ \\
\hline $\mathrm{C} 2-\mathrm{H} 2$ & 0.9300 & $\mathrm{C} 11-\mathrm{H} 11$ & 0.9300 \\
\hline $\mathrm{C} 3-\mathrm{C} 4$ & $1.371(10)$ & $\mathrm{C} 12-\mathrm{C} 13$ & $1.392(10)$ \\
\hline $\mathrm{C} 3-\mathrm{H} 3$ & 0.9300 & $\mathrm{C} 12-\mathrm{H} 12$ & 0.9300 \\
\hline $\mathrm{C} 4-\mathrm{C} 5$ & $1.357(10)$ & $\mathrm{C} 13-\mathrm{H} 13$ & 0.9300 \\
\hline $\mathrm{C} 4-\mathrm{Cl1}$ & $1.739(7)$ & $\mathrm{C} 14-\mathrm{H} 14 \mathrm{~A}$ & 0.9600 \\
\hline $\mathrm{C} 5-\mathrm{C} 6$ & $1.383(9)$ & $\mathrm{C} 14-\mathrm{H} 14 \mathrm{~B}$ & 0.9600 \\
\hline $\mathrm{C} 5-\mathrm{H} 5$ & 0.9300 & $\mathrm{C} 14-\mathrm{H} 14 \mathrm{C}$ & 0.9600 \\
\hline $\mathrm{C} 6-\mathrm{H} 6$ & 0.9300 & $\mathrm{~N} 1-\mathrm{S} 1$ & $1.651(5)$ \\
\hline $\mathrm{C} 7-\mathrm{O} 3$ & $1.192(7)$ & $\mathrm{N} 1-\mathrm{H} 1 \mathrm{~N}$ & $0.86(2)$ \\
\hline $\mathrm{C} 7-\mathrm{N} 1$ & $1.400(8)$ & $\mathrm{O} 1-\mathrm{S} 1$ & $1.426(4)$ \\
\hline $\mathrm{C} 7-\mathrm{C} 8$ & $1.520(9)$ & $\mathrm{O} 2-\mathrm{S} 1$ & $1.432(5)$ \\
\hline $\mathrm{C} 8-\mathrm{C} 13$ & $1.380(9)$ & $\mathrm{O} 4-\mathrm{H} 41$ & $0.84(2)$ \\
\hline $\mathrm{C} 8-\mathrm{C} 9$ & $1.398(9)$ & $\mathrm{O} 4-\mathrm{H} 42$ & $0.84(2)$ \\
\hline $\mathrm{C} 9-\mathrm{C} 10$ & $1.387(9)$ & & \\
\hline $\mathrm{C} 2-\mathrm{C} 1-\mathrm{C} 6$ & $120.5(6)$ & $\mathrm{C} 11-\mathrm{C} 10-\mathrm{C} 14$ & $120.9(7)$ \\
\hline $\mathrm{C} 2-\mathrm{C} 1-\mathrm{S} 1$ & $120.0(5)$ & $\mathrm{C} 9-\mathrm{C} 10-\mathrm{C} 14$ & $121.0(7)$ \\
\hline $\mathrm{C} 6-\mathrm{C} 1-\mathrm{S} 1$ & $119.5(5)$ & $\mathrm{C} 10-\mathrm{C} 11-\mathrm{C} 12$ & $122.1(7)$ \\
\hline $\mathrm{C} 1-\mathrm{C} 2-\mathrm{C} 3$ & $118.9(7)$ & $\mathrm{C} 10-\mathrm{C} 11-\mathrm{H} 11$ & 118.9 \\
\hline $\mathrm{C} 1-\mathrm{C} 2-\mathrm{H} 2$ & 120.6 & $\mathrm{C} 12-\mathrm{C} 11-\mathrm{H} 11$ & 118.9 \\
\hline $\mathrm{C} 3-\mathrm{C} 2-\mathrm{H} 2$ & 120.6 & $\mathrm{C} 11-\mathrm{C} 12-\mathrm{C} 13$ & $120.0(7)$ \\
\hline $\mathrm{C} 4-\mathrm{C} 3-\mathrm{C} 2$ & $120.1(7)$ & $\mathrm{C} 11-\mathrm{C} 12-\mathrm{H} 12$ & 120.0 \\
\hline $\mathrm{C} 4-\mathrm{C} 3-\mathrm{H} 3$ & 119.9 & $\mathrm{C} 13-\mathrm{C} 12-\mathrm{H} 12$ & 120.0 \\
\hline $\mathrm{C} 2-\mathrm{C} 3-\mathrm{H} 3$ & 119.9 & $\mathrm{C} 8-\mathrm{C} 13-\mathrm{C} 12$ & $118.6(7)$ \\
\hline $\mathrm{C} 5-\mathrm{C} 4-\mathrm{C} 3$ & $121.5(7)$ & $\mathrm{C} 8-\mathrm{C} 13-\mathrm{H} 13$ & 120.7 \\
\hline $\mathrm{C} 5-\mathrm{C} 4-\mathrm{Cl1}$ & $119.0(6)$ & $\mathrm{C} 12-\mathrm{C} 13-\mathrm{H} 13$ & 120.7 \\
\hline $\mathrm{C} 3-\mathrm{C} 4-\mathrm{Cl1}$ & $119.4(6)$ & $\mathrm{C} 10-\mathrm{C} 14-\mathrm{H} 14 \mathrm{~A}$ & 109.5 \\
\hline $\mathrm{C} 4-\mathrm{C} 5-\mathrm{C} 6$ & $119.4(7)$ & $\mathrm{C} 10-\mathrm{C} 14-\mathrm{H} 14 \mathrm{~B}$ & 109.5 \\
\hline $\mathrm{C} 4-\mathrm{C} 5-\mathrm{H} 5$ & 120.3 & $\mathrm{H} 14 \mathrm{~A}-\mathrm{C} 14-\mathrm{H} 14 \mathrm{~B}$ & 109.5 \\
\hline $\mathrm{C} 6-\mathrm{C} 5-\mathrm{H} 5$ & 120.3 & $\mathrm{C} 10-\mathrm{C} 14-\mathrm{H} 14 \mathrm{C}$ & 109.5 \\
\hline $\mathrm{C} 5-\mathrm{C} 6-\mathrm{C} 1$ & $119.7(6)$ & $\mathrm{H} 14 \mathrm{~A}-\mathrm{C} 14-\mathrm{H} 14 \mathrm{C}$ & 109.5 \\
\hline $\mathrm{C} 5-\mathrm{C} 6-\mathrm{H} 6$ & 120.2 & $\mathrm{H} 14 \mathrm{~B}-\mathrm{C} 14-\mathrm{H} 14 \mathrm{C}$ & 109.5 \\
\hline $\mathrm{C} 1-\mathrm{C} 6-\mathrm{H} 6$ & 120.2 & $\mathrm{C} 7-\mathrm{N} 1-\mathrm{S} 1$ & $122.9(4)$ \\
\hline $\mathrm{O} 3-\mathrm{C} 7-\mathrm{N} 1$ & $123.0(6)$ & $\mathrm{C} 7-\mathrm{N} 1-\mathrm{H} 1 \mathrm{~N}$ & $118(4)$ \\
\hline $\mathrm{O} 3-\mathrm{C} 7-\mathrm{C} 8$ & $123.7(6)$ & $\mathrm{S} 1-\mathrm{N} 1-\mathrm{H} 1 \mathrm{~N}$ & $114(4)$ \\
\hline $\mathrm{N} 1-\mathrm{C} 7-\mathrm{C} 8$ & $113.3(6)$ & $\mathrm{H} 41-\mathrm{O} 4-\mathrm{H} 42$ & $113(9)$ \\
\hline $\mathrm{C} 13-\mathrm{C} 8-\mathrm{C} 9$ & $120.4(6)$ & $\mathrm{O} 1-\mathrm{S} 1-\mathrm{O} 2$ & $119.5(3)$ \\
\hline $\mathrm{C} 13-\mathrm{C} 8-\mathrm{C} 7$ & $124.2(6)$ & $\mathrm{O} 1-\mathrm{S} 1-\mathrm{N} 1$ & $108.8(3)$ \\
\hline $\mathrm{C} 9-\mathrm{C} 8-\mathrm{C} 7$ & $115.4(6)$ & $\mathrm{O} 2-\mathrm{S} 1-\mathrm{N} 1$ & $104.8(3)$ \\
\hline $\mathrm{C} 10-\mathrm{C} 9-\mathrm{C} 8$ & $120.7(6)$ & $\mathrm{O} 1-\mathrm{S} 1-\mathrm{C} 1$ & $109.8(3)$ \\
\hline $\mathrm{C} 10-\mathrm{C} 9-\mathrm{H} 9$ & 119.7 & $\mathrm{O} 2-\mathrm{S} 1-\mathrm{C} 1$ & $107.9(3)$ \\
\hline $\mathrm{C} 8-\mathrm{C} 9-\mathrm{H} 9$ & 119.7 & $\mathrm{~N} 1-\mathrm{S} 1-\mathrm{C} 1$ & $105.2(3)$ \\
\hline
\end{tabular}




$\begin{array}{llll}\mathrm{C} 11-\mathrm{C} 10-\mathrm{C} 9 & 118.1(7) & & \\ \mathrm{C} 6-\mathrm{C} 1-\mathrm{C} 2-\mathrm{C} 3 & -1.4(11) & \mathrm{C} 9-\mathrm{C} 10-\mathrm{C} 11-\mathrm{C} 12 & 1.5(11) \\ \mathrm{S} 1-\mathrm{C} 1-\mathrm{C} 2-\mathrm{C} 3 & 177.0(6) & \mathrm{C} 14-\mathrm{C} 10-\mathrm{C} 11-\mathrm{C} 12 & -179.5(7) \\ \mathrm{C} 1-\mathrm{C} 2-\mathrm{C} 3-\mathrm{C} 4 & 0.5(12) & \mathrm{C} 10-\mathrm{C} 11-\mathrm{C} 12-\mathrm{C} 13 & -2.1(12) \\ \mathrm{C} 2-\mathrm{C} 3-\mathrm{C} 4-\mathrm{C} 5 & 0.2(13) & \mathrm{C} 9-\mathrm{C} 8-\mathrm{C} 13-\mathrm{C} 12 & 0.5(11) \\ \mathrm{C} 2-\mathrm{C} 3-\mathrm{C} 4-\mathrm{C} 11 & -178.5(6) & \mathrm{C} 7-\mathrm{C} 8-\mathrm{C} 13-\mathrm{C} 12 & 178.7(6) \\ \mathrm{C} 3-\mathrm{C} 4-\mathrm{C} 5-\mathrm{C} 6 & 0.0(12) & \mathrm{C} 11-\mathrm{C} 12-\mathrm{C} 13-\mathrm{C} 8 & 1.0(12) \\ \mathrm{C} 11-\mathrm{C} 4-\mathrm{C} 5-\mathrm{C} 6 & 178.6(5) & \mathrm{O} 3-\mathrm{C} 7-\mathrm{N} 1-\mathrm{S} 1 & -2.9(9) \\ \mathrm{C} 4-\mathrm{C} 5-\mathrm{C} 6-\mathrm{C} 1 & -0.8(11) & \mathrm{C} 8-\mathrm{C} 7-\mathrm{N} 1-\mathrm{S} 1 & 177.3(4) \\ \mathrm{C} 2-\mathrm{C} 1-\mathrm{C} 6-\mathrm{C} 5 & 1.5(10) & \mathrm{C} 7-\mathrm{N} 1-\mathrm{S} 1-\mathrm{O} 1 & 46.8(6) \\ \mathrm{S} 1-\mathrm{C} 1-\mathrm{C} 6-\mathrm{C} 5 & -176.9(5) & \mathrm{C} 7-\mathrm{N} 1-\mathrm{S} 1-\mathrm{O} 2 & 175.7(5) \\ \mathrm{O} 3-\mathrm{C} 7-\mathrm{C} 8-\mathrm{C} 13 & -159.8(7) & \mathrm{C} 7-\mathrm{N} 1-\mathrm{S} 1-\mathrm{C} 1 & -70.7(5) \\ \mathrm{N} 1-\mathrm{C} 7-\mathrm{C} 8-\mathrm{C} 13 & 19.9(9) & \mathrm{C} 2-\mathrm{C} 1-\mathrm{S} 1-\mathrm{O} 1 & -27.6(6) \\ \mathrm{O} 3-\mathrm{C} 7-\mathrm{C} 8-\mathrm{C} 9 & 18.5(9) & \mathrm{C} 6-\mathrm{C} 1-\mathrm{S} 1-\mathrm{O} 1 & 22.2(6) \\ \mathrm{N} 1-\mathrm{C} 7-\mathrm{C} 8-\mathrm{C} 9 & -161.7(5) & \mathrm{C} 2-\mathrm{C} 1-\mathrm{S} 1-\mathrm{O} 2 & -159.4(5) \\ \mathrm{C} 13-\mathrm{C} 8-\mathrm{C} 9-\mathrm{C} 10 & -1.0(10) & \mathrm{C} 6-\mathrm{C} 1-\mathrm{S} 1-\mathrm{O} 2 & -89.2(6) \\ \mathrm{C} 7-\mathrm{C} 8-\mathrm{C} 9-\mathrm{C} 10 & -179.4(6) & \mathrm{C} 2-\mathrm{C} 1-\mathrm{S} 1-\mathrm{N} 1 & 89.2(6) \\ \mathrm{C} 8-\mathrm{C} 9-\mathrm{C} 10-\mathrm{C} 11 & 0.1(10) & \mathrm{C} 6-\mathrm{C} 1-\mathrm{S} 1-\mathrm{N} 1 & \\ \mathrm{C} 8-\mathrm{C} 9-\mathrm{C} 10-\mathrm{C} 14 & -179.0(6) & & \end{array}$

Hydrogen-bond geometry $\left(A,{ }^{\circ}\right)$

\begin{tabular}{lllll}
\hline$D-\mathrm{H} \cdots A$ & $D-\mathrm{H}$ & $\mathrm{H} \cdots A$ & $D \cdots A$ & $D-\mathrm{H} \cdots A$ \\
\hline $\mathrm{N} 1-\mathrm{H} 1 N \cdots \mathrm{O} 4$ & $0.86(2)$ & $1.93(2)$ & $2.771(8)$ & $169(6)$ \\
$\mathrm{O} 4-\mathrm{H} 41 \cdots \mathrm{O} 1^{\mathrm{i}}$ & $0.84(2)$ & $2.29(7)$ & $2.916(7)$ & $131(8)$ \\
$\mathrm{O} 4-\mathrm{H} 41 \cdots \mathrm{O} 3^{\mathrm{i}}$ & $0.84(2)$ & $2.42(6)$ & $3.117(8)$ & $140(8)$ \\
$\mathrm{O} 4-\mathrm{H} 42 \cdots \mathrm{O} 2^{\mathrm{ii}}$ & $0.84(2)$ & $2.35(6)$ & $3.022(8)$ & $137(8)$ \\
\hline
\end{tabular}

Symmetry codes: (i) $-x+1 / 2, y+1 / 2, z$; (ii) $x+1, y, z$. 